\title{
Practice educators' attitudes and perspectives of interprofessional collaboration and interprofessional practice learning for students: a mixed-methods case study
}

\author{
O'Carroll, Veronica, McSwiggan, Linda, Campbell, Martin
}

\begin{tabular}{|l|l|}
\hline Date of deposit & 29112018 \\
\hline Document version & Author's accepted manuscript \\
\hline Access rights & $\begin{array}{l}\text { C } 2018, \text { Taylor \& Francis/Informa. This work is made available } \\
\text { online in accordance with the publisher's policies. This is the } \\
\text { author created, accepted version manuscript following peer } \\
\text { review and may differ slightly from the final published version. }\end{array}$ \\
\hline $\begin{array}{l}\text { Citation for } \\
\text { published version }\end{array}$ & $\begin{array}{l}\text { O'Carroll, V., McSwiggan, L., and Campbell, M. (2018). Practice } \\
\text { educators' attitudes and perspectives of interprofessional } \\
\text { collaboration and interprofessional practice learning for students: } \\
\text { a mixed-methods case study. Journal of Interprofessional Care, } \\
\text { Latest articles }\end{array}$ \\
\hline $\begin{array}{l}\text { Link to published } \\
\text { version }\end{array}$ & \begin{tabular}{l} 
https://doi.org/10.1080/13561820.2018.1551865 \\
\hline
\end{tabular} \\
\hline
\end{tabular}

Full metadata for this item is available in St Andrews Research

Repository at: https://research-repository.st-andrews.ac.uk/

\section{St Andrews Research Repository}




\section{Abstract}

Interprofessional collaboration (IPC) is important for the delivery of effective integrated health and social care systems. Interprofessional practice learning (IPPL) enables students to learn to work together within a relevant context and prepare for future IPC. Whilst there is some evidence that negative attitudes impact on IPC and interprofessional education, there is a dearth of research on health and social work professionals' attitudes and perspectives of IPC, and IPPL opportunities for students. A mixed-methods case study was used to investigate practice educators' attitudes and perspectives of IPC, and IPPL for their students. Results showed that attitudes were positive and that mainly meso and macro level factors, as opposed to micro level, impacted on the implementation of IPC, and IPPL for students' learning in practice settings. IPC was perceived to be best enabled by effective communication, established teams, IPPL for staff, and shared processes and policies. Close working proximity to other professionals encouraged informal communication and positive interprofessional relationships. Motivation and resources were perceived as enablers of IPPL but there were often missed opportunities for IPPL. These findings suggest that further work is required to identify systems for improving IPC and to enhance IPPL opportunities for students learning within practice settings.

\section{Introduction}

As health and social care services have integrated, there is an increased need for more effective interprofessional collaboration (IPC) amongst health and social care professionals. Alignment between practice settings and higher education institutes, and the support of practice educators who are responsible for facilitating students' learning in practice is vital for maintaining the continuum of IPE 
into practice (Institute of Medicine [IOM]; 2015; Barr \& Low, 2013; CAIPE, 2002;

WHO, 2010). Practice settings offer the opportunity for accessible interprofessional practice learning (IPPL) for students (O'Carroll, Braid, Jackson \& Ker, 2012;

O'Carroll, Smith, Collinson, Jackson \& Ker, 2013). However, opportunities for IPPL are often untapped (IOM, 2015; Pollard, Miers \& Rickaby, 2012; Stew, 2005) and experiences of effective IPC can be varied (Stew, 2005; Pollard et al.2012). The value that practice educators place on students' experiences of IPC and IPPL is therefore of importance.

\section{Background}

A review of the literature relating to attitudes to IPC and IPE (O'Carroll, McSwiggan \& Campbell, 2016) identified three main research gaps in empirical research to date. These gaps included: the limited number of studies which focused on the attitudes of both health care and social work professionals to IPC, and IPPL specifically for students; the limited evidence of variables affecting attitudes; and limited insight into practice educators' perspectives of the enablers and barriers to IPC, and IPPL for students. Since this review, a pilot study by Ong, Tan, Knab, Farrell and Lim (2017) investigated practice educators' attitudes towards IPC and IPPL. There is still limited understanding however, of practice educators' perspectives of the enablers and barriers to IPC, and IPPL for their students. These limitations provided a clear rationale for undertaking further empirical work in this area.

This paper reports on two objectives from an unpublished doctoral thesis (O'Carroll, 2017). These objectives were: to measure practice educators' attitudes to IPC, and their attitudes towards IPPL for their students, and to explore their perspectives of the enablers and barriers to IPC and IPPL in practice settings. 
Context and perspective were key to the aim and scope of the study. Firstly, it was carried out within the context of practice settings, where health care and social work students learn as part of their training. Secondly, the attitudes and perspectives of a range of health care and social work professionals, specifically professionals who mentor or supervise students during their practice placements, were sought.

\section{Methods}

Research Design

A mixed-methods single case study design was used for an in-depth investigation of participants' perceptions and experiences, using an array of evidence (Creswell, 2015; Gillham, 2010; Yin 2014). As a single case study, it was anticipated that the study would "shed light on a larger class of cases" (Gerring, 2007, p.20) and add to existing knowledge and experience (Stake, 1995; Yin, 2014). To measure practice educators' attitudes towards IPC and IPPL, an online survey was used to collect quantitative data. A qualitative approach was used to explore practice educators' perspectives of the enablers and barriers of IPC and IPPL through semi-structured, face-to-face interviews. As a core quantitative study with qualitative follow-up contribution (QUANT $\longrightarrow$ qual), this sequence of data collection is in keeping with Creswell's (2015) definition of an explanatory sequential design; the data gained from one method (semi-structured interviews) was used to explicate the data generated from another method (surveys).

The research was undertaken within one health board and local authority in Scotland. The health board consisted of one main hospital offering acute in-patient services, eight community hospitals providing outpatient and rehabilitation services, and 71 health centres. The Local authority works in partnership with the health 
board in their provision of services to children and young families, and young and older adults.

\section{Data Collection}

Data was collected between December 2013 and April 2015. Following an initial period of quantitative data collection via the online survey, the semi-structured interviews commenced in February 2014. Multi stage, non-random sampling strategies; criterion-i, quota sampling and snowball sampling were used to recruit professionals with a specific remit in mentoring or supervising health or social work students during practice placements. The interview sample was drawn from survey participants who indicated their agreement to be interviewed. To enhance the comparative potential of the interview data set, a broad range of participants were purposively sampled to ensure that a range of characteristics were included.

Curran, Sharpe and Forristall's (2007) adapted version of Parsell and Bligh's (1999) the Readiness for Interprofessional Learning Scale (RIPLS) and Kenaszchuk, Reeves, Nicholas and Zwarenstein (2010) Multigroup Measurement Scale, adapted from Adams, Bond and Arber (1995), were used, with permission, to measure attitudes towards IPC and IPPL. Kenaszchuk et al. (2010) identified the three subscales of communication, accommodation and isolation in the Multigroup Measurement Scale. Curran et al. (2007) adapted the RIPLS by removing some of the items to create a 15-item scale. They maintained the three factors; teamwork and collaboration, professional identity, and roles and responsibilities. They also amended the wording of each item so that it was relevant to tutors with a role in teaching health care students, and so that their attitudes towards IPE for students could be investigated. These pre-validated scales were combined to create a survey 
relevant to all professionals who mentor or supervise health care or social work students during practice placements.

In their version of the Multigroup Measurement Scale, Kenaszchuk et al. (2010) adopted a 4-point Likert scale which differed from previous 5-point scales applied to Curran et al's adapted RIPLS. To encourage more definitive responses and avoid a high rate of neutral responses (Sullivan-Bollyai \& Grey, 2002), the survey used in this study adopted a 4-point Likert scale (1=strongly disagree to 4=strongly agree). This scoring was reversed for negatively orientated questions. Scores above two for each item indicated positive attitudes towards IPC or IPPL.

The reliability of the survey in its combined form was measured using a testre-test method to measure intra-rater reliability. This was undertaken with a separate sample of participants from another health board and local authority. A Kappa value of 0.42 confirmed that the survey had moderate test-retest reliability and was acceptable to use for the purposes of this study.

Implied consent was obtained by completion of the survey. Demographic information collected within the survey included: participants' professional group, governing body, gender, years of experience, and previous experience of IPE. The final section of the survey enabled participants to opt in or out of the semi-structured interviews. In addition, a free text section was included to provide the opportunity for some participants, who opted out of the interviews, to provide some additional qualitative data.

Face-to-face semi-structured interviews were undertaken with a purposeful sample of survey participants who agreed to be interviewed. An interview topic guide (Table 1) was utilised to encourage a focused yet open approach to data generation, whilst ensuring that the discussion aligned to the study objectives. 
Written consent was gained from the participants and interviews were carried out mainly at participants' workplaces. The interviews lasted 30-40 minutes. They were audio recorded and transcribed verbatim by the first author and a research assistant.

Table 1. Semi structured interview topic guide

\section{Opening statement}

Opening question

Guide topics as discussion unfolds
You recently completed an online survey related to IPC, and IPPL for the students you supervise in practice settings. As a reminder of what these terms mean, IPC is related to different professionals working collaboratively together in practice. IPPL is related to students from different professions learning together in the practice placement setting.

Can you tell me a little bit about where your work currently and which professionals you usually work with on a regular basis?

- Enablers of IPC in the practice setting

- Barriers to IPC in the practice setting

- Opportunities in the practice placement setting for students to be involved in IPPL

- Enablers of IPPL in the practice placement setting

- Barriers to IPPL in the practice placement setting

- Previous experiences of IPE as either a facilitator or as a learner

Data Analysis

The quantitative data was analysed to summarise the characteristics of the sample and to provide mean scores from the survey. The framework approach, as described by Ritchie and Spencer (1994) guided the analysis of the qualitative data. A deductive approach was initially used to identify data related to enablers and barriers of IPC and IPPL, followed by an inductive method to further identify emerging primary and secondary sub-themes. A research assistant reviewed a sample of the sub-themes for verification. 


\section{Ethical Considerations}

Ethical approval was obtained from the University of St Andrews and permission was given by the health board and local authority to access the study sites. No incentives were offered for participation.

\section{Results}

Practice Educators' Attitudes towards IPC and IPPL

As shown in Table 2, a total of 90 practice educators completed the survey with the largest number of responses from professionals governed by the Nursing and Midwifery Council (NMC). According to a workforce report published in 2012 by the health board included in this study, it was reported that clinical and non-clinical professionals comprised of $15.6 \%$ males and $84.4 \%$ females with a mean age of 44 . The sample in this study was therefore fairly representative, in terms of gender and years of experience; over half of the sample were female $(66.7 \%)$ and $31.1 \%$ of participants were qualified for more than 25 years. As shown in Table 2, 47.8\% worked within a community setting. This may reflect the large number of community hospitals (8) and health care centres (71) in the region of the health board. In relation to prior experience of IPE, $78.9 \%$ of participants reported that they had prior experience of IPE and $42.2 \%$ reported that this experience was both as an educator and as a learner. Table 3 provides a breakdown of the range of professionals interviewed. 
Table 2. Demographic profile of the survey and interview participants

\begin{tabular}{llcc}
\hline & & $\begin{array}{c}\text { Survey participants } \\
\text { Total: } \mathbf{n = 9 0} \\
\mathbf{n}(\%)\end{array}$ & $\begin{array}{c}\text { Interview } \\
\text { participants } \\
\text { Total: } \mathbf{n = 2 2}\end{array}$ \\
\hline Governing Body & $\mathrm{NMC}^{1}$ & $35(38.9)$ & $10(45.4)$ \\
& $\mathrm{GMC}^{2}$ & $22(24.4 \%)$ & $4(18.2)$ \\
& $\mathrm{HCPC}^{3}$ & $21(23.3 \%)$ & $5(22.7)$ \\
& $\mathrm{SSSC}^{4}$ & $9(10 \%)$ & $2(9.1)$ \\
Gender & $\mathrm{GPhC}^{5}$ & $3(3.3 \%)$ & $1(4.5)$ \\
& Female & $60(66.7 \%)$ & $15(68.2)$ \\
Area of work & Male & $30(33.3 \%)$ & $7(31.8)$ \\
& Community & $43(47.8 \%)$ & $12(54.5)$ \\
& Acute & $30(33.3 \%)$ & $5(22.7)$ \\
Years' qualified & Both community and & $8(8.9 \%)$ & $3(13.6)$ \\
& Local authority & $9(10 \%)$ & $2(9.1)$ \\
& $1-10$ years & $17(18.9 \%)$ & $4(18.2)$ \\
& $11-15$ years & $21(23.3 \%)$ & $1(4.5)$ \\
Previous experience & $11(12.2 \%)$ & $2(9.1)$ \\
of IPE & $16-20$ years & $13(14.4 \%)$ & $7(31.8)$ \\
Type of IPE & Yes & $28(31.1 \%)$ & $8(36.4)$ \\
Experience & Yore than 25 years & $71(78.9 \%)$ & $18(81.8)$ \\
& No & $14(15.5 \%)$ & $2(9.1)$ \\
& Unsure & $5(5.5 \%)$ & $2(9.1)$ \\
& Learner & $20(22.2 \%)$ & $5(22.7)$ \\
& Educator & $13(14.4 \%)$ & $4(18.2)$ \\
& Both & $38(42.2 \%)$ & $9(40.9)$ \\
& Neither & $14(15.5 \%)$ & $2(9.1)$ \\
& Unsure & $5(5.5 \%)$ & $2(9.1)$ \\
\hline
\end{tabular}

1. Nursing and Midwifery Council

2. General Medical Council

3. Health and Care Professions Council

4. Scottish Social Services Council

5. General Pharmaceutical Council 
Table 3. Interview participants' professional groups

\begin{tabular}{|c|c|c|c|c|}
\hline GMC & HCPC & NMC & SSSC & GPhC \\
\hline $\mathrm{GP}(n=3)$ & $\begin{array}{l}\text { Clinical } \\
\text { Psychologist }(n=1)\end{array}$ & Adult Nurse $(n=1)$ & Social Worker $(n=2)$ & Pharmacist $(n=1)$ \\
\hline \multirow[t]{6}{*}{$\begin{array}{l}\text { Medical Consultant } \\
(n=1)\end{array}$} & $\begin{array}{l}\text { Occupational } \\
\text { Therapist }(n=1)\end{array}$ & District Nurse $(n=3)$ & & \\
\hline & $\begin{array}{l}\text { Physiotherapist } \\
(n=1)\end{array}$ & Health Visitor $(n=2)$ & & \\
\hline & Radiographer $(n=1)$ & $\begin{array}{l}\text { Mental Health } \\
\text { Nurse }(n=2)\end{array}$ & & \\
\hline & Dietician $(n=1)$ & & & \\
\hline & & Midwife $(n=1)$ & & \\
\hline & & $\begin{array}{l}\text { Learning } \\
\text { Disabilities Nurse } \\
(n=1)\end{array}$ & & \\
\hline Total: $n=4(18.2 \%)$ & Total: $n=5(22.7 \%)$ & Total $n=10(45 \%)$ & Total: $n=2(9.1 \%)$ & Total $n=1(4.5 \%)$ \\
\hline
\end{tabular}

As mentioned earlier, the survey used in this study consisted of two prevalidated scales; Curran, Sharpe and Forristall's (2007) adapted Readiness for Interprofessional Learning Scale (Parsell \& Bligh, 1999) and Kenaszchuk, Reeves, Nicholas and Zwarenstein's (2010) Multigroup Measurement Scale. Table 4 provides an overview of the mean sub-scale scores for each of these scales. In the Multigroup Measurement Scale, the total mean sub-scale scores for communication $(M=2.6[S D=0.4])$, accommodation $(M=2.7[S D=0.4]$ and isolation $(M=2.8[S D=0.4])$ indicated that practice educators' attitudes towards IPC were positive. In the adapted RIPLS, the mean sub-scale scores for teamwork and collaboration $(M=3.3$ $[S D=0.4])$ and professional identity $(M=3.2[S D=0.4])$ indicated that practice educators' attitudes towards IPPL for their students were positive. 
Table 4. Attitudes to IPC and IPPL mean scores

\begin{tabular}{|c|c|c|c|c|c|}
\hline \multicolumn{4}{|c|}{ Attitudes to IPC (Multigroup Measurement Scale) } & \multicolumn{2}{|c|}{ Attitudes to IPPL (RIPLS) } \\
\hline & Communication & Accomodation & Isolation & $\begin{array}{l}\text { Teamwork \& } \\
\text { Collaboration }\end{array}$ & $\begin{array}{l}\text { Professional } \\
\text { Identity }\end{array}$ \\
\hline NMC $(n=35)$ & 2.6 & 2.6 & 2.8 & 3.3 & 3.2 \\
\hline GMC $(n=22)$ & 2.7 & 2.7 & 2.9 & 3.2 & 3.1 \\
\hline $\operatorname{HCPC}(n=21)$ & 2.6 & 2.8 & 2.8 & 3.4 & 3.2 \\
\hline $\operatorname{SSSC}(n=9)$ & 2.6 & 2.9 & 2.6 & 3.1 & 2.9 \\
\hline GPhC $(n=3)$ & 2.7 & 3.0 & 2.7 & 3.7 & 3.3 \\
\hline Total $M$ and $S D$ & $\begin{array}{c}M=2.6 \\
(S D=0.4)\end{array}$ & $\begin{array}{c}M=2.7 \\
(S D=0.4)\end{array}$ & $\begin{array}{c}M=2.8 \\
(S D=0.4)\end{array}$ & $\begin{array}{c}M=3.3 \\
(S D=0.4)\end{array}$ & $\begin{array}{c}M=3.2 \\
(S D=0.4)\end{array}$ \\
\hline
\end{tabular}

Enablers and Barriers of IPC

Four primary sub-themes related to enablers of IPC emerged from the qualitative data (Figure 1). These were: effective communication, established teams, IPPL for qualified professionals, and shared processes and policies. Contrasting enablers and barriers of IPC were identified: effective and ineffective communication, established and transient teams, and shared versus differing processes and policies. Sub-themes which emerged as unique to the overarching theme of barriers of IPC included: organisational culture, lack of resources, and lack of understanding of roles and responsibilities. 


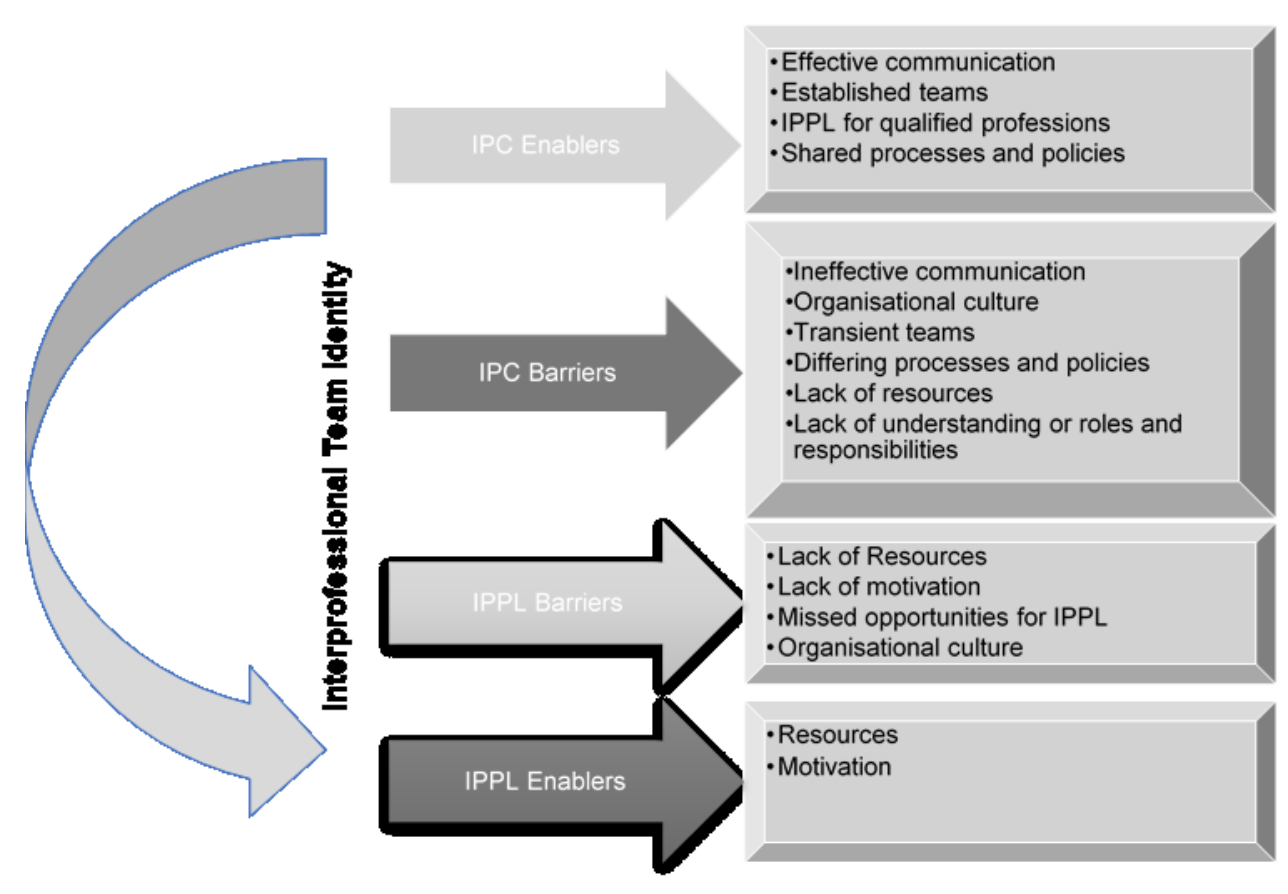

Effective and Ineffective Communication

Effective communication was facilitated by regular planned meetings such as interprofessional meetings and safety briefings involving a number of different professionals. However, there was the tendency for other work priorities to take precedence over these planned meetings:

"It could fall into the category of preventative, prophylactic type work... but often goes on the wayside if you have got a lot of other things on and then you don't have time for that." (Social Worker, local authority, interview 22)

Lack of opportunity for regular structured meetings was perceived as a missed opportunity to keep up-to-date with plans of care for service users and to strengthen relationships within the team. The absence of regular meetings was particularly challenging in community care contexts. It made it difficult to coordinate care amongst and range of different professionals and to maintain contact between health care and social work: 
"We don't have multidisciplinary meetings, you know, if there is a multidisciplinary meeting it's for a specific child, it's not something we would do as a group, just to share relationships or anything like that." (Health Visitor, community, interview 9)

Unplanned, ad hoc instances of communication were viewed as complementary to planned communication. Participants described opportunistic discussions and informal referrals which occurred, for example, "in passing" or during coffee breaks. Shared work spaces, which brought a range of professionals into close working proximity within one department, such as open plan offices, enabled interprofessional communication. Staff rooms and joint coffee breaks were frequently mentioned as providing a social area as well as an opportunity to informally discuss work issues:

"Just from a social point of view, everyone gets together in the coffee room ...we'll often discuss cases with people we are not quite sure what to do with over coffee." (GP, community, interview 2)

In contrast, some participants highlighted the difficulties of noise levels and lack of privacy in open plan settings. However, these negative points seemed to be outweighed by the value of being able to get to know other members of the team on a more personal level:

"We are now in open plan which with it brings all its issues, but in reality, what has been good...is that I now know what the criminal justice workers look like....and for that we are becoming real people to each other....it comes down to person, being a person first and then being a professional second." (Social worker, local authority, interview 21)

Where close working proximity was not evident, participants stated that they often found that it was difficult "to get hold of" other professionals. This seemed to be particularly challenging within the community setting where it was highlighted that professionals were moving between different locations on a day-to-day basis: 
"I think with social work especially... [it is] so hard to get people in and you end up ping-ponging phone calls back-and-forwards for days sometimes, or even a couple of weeks before you actually manage to get the person that you've been trying to get." (Health Visitor, community, interview 9)

A number of participants within community care contexts who had been relocated due to centralising of health and social care services, described feelings of isolation, loss, and resentment. There was a sense of grieving for their smaller intimate teams that they had been part of, prior to their relocation:

"It wasn't our own home either, we felt we were visitors in a bigger organisation and we also felt that we were getting dragged into NHS screaming and kicking, no longer part of the practice team...it was a wrench for us all of us." (District Nurse, community, interview 6)

Moving to a location where there was no staff room was perceived to have an additional impact on team identity and confounded feelings of loss:

"Before, we all intermingled in the same coffee room. Here, we were told that we weren't allowed to have a coffee room, we all had to use the canteen and there are conversations we have at lunch times that are not suitable for public consumption and you know we've lost the friendly chit chat with other people." (District Nurse, community, interview 5)

Electronic communication included systems such as email, online referral systems, instant messaging and electronic records. Instant messaging systems were valued as an effective and efficient way of alerting colleagues to non-urgent issues. However, it was also noted these messages, at times, disrupted clinics as participants were conscious of receiving messages during consultations. Electronic systems were reported to only work in some areas of a building or were not used consistently by all staff. Inability to share or access some electronic records between health, social care and police was reported as having a negative impact on communication of key information, particularly in relation to child protection issues: 
"Other areas are getting all these things through from the police and we get very little so it's definitely electronic systems that work and the child protection one, there is things going on that we are very aware of that we are not hearing about." (Health Visitor, community, interview 8)

Frustrations were expressed where communication was perceived to be one sided. Some participants reported that they would often initiate communication with other professionals to update them on service users' progress but rarely had this reciprocated. Communication difficulties were particularly noted between health and social care professionals during weekends:

'We've had a lot of the patients at the weekend admitted to the hospital. Home care' would've known that because it's the weekend, but nobody passed the information on to us". (District Nurse, community, interview 7)

\section{Lack of Resources}

Lack of resources emerged as a large sub-theme of barriers to IPC. Money constraints, lack of time, lack of leadership, and varying budgets between different professionals were highlighted as some of the main resource barriers. Some of the coping strategies to manage these resource issues included non-attendance at planned interprofessional team meetings and referring on to other professionals. They were perceived as impacting negatively on IPC.

"Realistically we don't have that resource and the referrals keep going up and we have to say no we can't take everybody and that's what creates the tensions." (Clinical Psychologist, acute and community, interview 18)

\section{Organisational Culture and IPC}

Although hierarchies and power differentials were discussed by some of the participants, hierarchical working structures were not always highlighted as specifically existing between professionals. Some of the accounts given by 
participants suggested that intraprofessional hierarchies existed within professional groups. Divisions between different care contexts were noted by one participant who referred to tensions between physicians in the community setting and in the acute setting:

"Within the last 5 years, there is a lot more us and them, secondary care [acute setting] and primary care [community setting] ..." (GP, community, interview 3)

For some, the issues were less complex than hierarchies as a barrier to IPC, and more related to individual personalities that impacted on team dynamics:

"You will come [across] at some point, some people who don't want to be flexible... they're quite dogmatic about the approach and prescriptive with the people that they're dealing with." (Health Visitor community, interview 9)

\section{Established and Transient Teams}

The terms "established teams" and "strong teams" were frequently mentioned by participants who identified that they had worked in interprofessional teams for several years. Particularly within the community setting, it was noted that established teams had good knowledge of their service users. Many participants maintained that this was invaluable for ensuring continuity in care delivery, where input from multiple professionals was required. With established teams, came awareness and understanding of each other's' roles and responsibilities, appreciation and mutual respect and a sense of interprofessional team identity. One participants provided an example of their colleagues from other professionals knocking on their door to seek advice or to get a second opinion. Conflict within established teams was perceived more easily resolved within teams that identified themselves as being well established: 
"They work out how your character works, you work out how their character works. You realise where there can be areas of conflict. You know how to steer around those areas of conflict...." (GP, community, interview 3)

In transient teams, participants highlighted issues such as referrals being made inappropriately or inaccessibility of other professionals' services. This seemed to be related to a lack of awareness of the availability of services available in some areas, being unfamiliar with how others' roles and responsibilities differed across different contexts, and being a 'visitor' to an established team.

\section{Interprofessional Practice Learning for Qualified Professionals}

A number of participants emphasised that being involved in joint training with other professionals enabled IPC. This training was also valued as a way of enabling ongoing IPC after the event and was utilised to build a network of professionals. In the main, such training activities were described as interprofessional in their delivery:

"Being able to get together in a less formal environment and talk about the different roles people do is really very helpful... it just felt like a really nice environment to demystify what each other did without any particular sensitivities." (Clinical Psychologist, acute and community, interview 18)

These IPPL activities seemed to occur where participants described a number of other IPC enablers. In these practice settings, there was a range of professionals working in close proximity, planned and unplanned communication was reported to have occurred and there was a sense of an interprofessional team identity. As illustrated in Figure 1, these findings suggest a possible link between IPPL, effective communication, proximity and established teams. 
Lack of Understanding of Roles and Responsibilities

This theme also linked closely to the theme of transient teams, as participants described uncertainty related to some roles and responsibilities within new teams. Integration of health and social care and new services to provide more care in the community brought new tensions because of overlaps in roles and responsibilities and uncertainty in role boundaries roles within similar professionals, and across health and social care services:

"There's always conflict on who's going to do what between nursing staff and social work staff and I think nursing staff are expected to do quite a lot more whereas we may see it as a social work job." (Mental Health Nurse, community, interview 10)

\section{Shared Versus Differing Processes and Policies}

The Getting It Right for Every Child (GIRFEC) national framework, for the implementation of the Children and Young People (Scotland) Act (2014), was referred to by a number of participants as an example of a shared policy which guided a range of professionals' practice in the community, acute and local authority settings. This was perceived as making an important contribution to enabling IPC and encouraged a coordinated approach between health, social care and other agencies, such as education and police:

'We are all protecting children and you've just got slightly different roles in that. The more we communicate and understand each other's roles, and we are all using the same paperwork and format now, then that can only be a good thing." (Health Visitor, community, interview 8)

Differing processes and policies used by professionals were reported as a barrier to IPC by several participants. Linked to this were concerns related to the integration and possible issues arising around professional governance. A pharmacist raised 
the issue of differing policies governing the administration of medicines in the community setting:

"They've [the health care system] have actually got health care assistants coming out administering medicines, which they perfectly can do, but we've got band 3 carers in the social care system who don't...so you see the potential friction coming in." (Pharmacist, acute, interview 17)

As well as changes in processes and policies, the perceived impact that the integration of health and social care had on line management and leadership was raised as an issue. Although changes had already been implemented, the perceived enormity of the impact of these changes instilled feelings of anxiety and uncertainty of how IPC could be achieved:

"Now we have different policies and procedures, different leadership, everything is different, it worries me that, that coming together. I don't know where we sit with that." (Adult Nurse, community, interview 12)

Some participants provided examples of differing referral systems between health care professionals and between health and social care, with some accepting face-to -face and others stipulating written referrals. These were highlighted as barriers to achieving effective IPC:

"Unfortunately, a lot of our colleagues need the piece of paper... it's not them, it's the system that they work in and the organisation that they work for." (District Nurse, community, interview 6)

\section{Enablers and Barriers of IPPL}

As shown in Figure 1, two contrasting sub-themes of resources and motivation were found in relation to enablers and barriers to IPPL. The subthemes of missed opportunities for IPPL, and organisational culture were specific to barriers to IPPL. Of all participants who were interviewed, there were only 
three who reported that they regularly facilitated IPPL activities for students, which also involved service users. These participants also shared positive experiences of IPC and identified themselves as working in an effective interprofessional team:

"We have such a good relationship with the charge nurse and nurses on the wards... so I just come in and say, 'Are there any students'? and they'll say, 'Here you are, off you go.' " (GP, community, interview 3)Motivation

The participants who had experience of facilitating IPPL for students highlighted that their colleagues' motivation was an enabler for these IPPL activities. This motivation also stemmed from reciprocal agreements within teams to support students learning within the practice setting and mutual gains from IPPL:

"There's a kind of reciprocal agreement, like the health visitors will say, 'Can you take one of our students out for the day?' " (Dietician, community and acute, interview 19)

Issues around the confidence of practice educators in organising IPPL, and teaching students from other professional groups was acknowledged as a potential barrier:

"I think often they (practice educators) ... are worried about getting people together on an ad hoc basis or are uncertain about how to approach interprofessional learning if they have no experience of that professional body." (GP, community, interview 2)

Some of the participants who had no experience of facilitating IPPL shared their uncertainty of the value of IPPL over other learning opportunities for students, and concern that it would add to students' learning load:

"I don't know how effective that (IPPL) would be, particularly when both sets of students have got a lot on their plate." (Mental Health Nurse, Community, interview 10) 
Doubt was also expressed of the added benefit of IPPL over students' experiences of IPE within the academic setting:

"All the students when at Uni are very used to doing IPE you know, that's hard to parcel, that's nothing new." (Physiotherapist, acute, interview 16)

\section{Resources}

Larger teams working in close proximity with other professionals capitalised on the ability to access each other and each other's students:

"We do work with CPNs, so they are in the building and the district nurses are in the building.... physios come in, OTs come in. We have various psychiatric services so there is a wide variety of opportunities there for our students." (Health Visitor, community, interview 9)

Where regular IPPL activities occurred, an IPE coordinator was perceived as a key resource particularly to identify when students shared the same placement:

"If that [input from the IPE coordinator] wasn't there...I don't think it would happen. It would be too much an obstacle." (District Nurse, community, interview 8)

Guaranteeing an interprofessional mix of students was highlighted as a challenge due to students "coming and going" at different times:

'We'll have a student for six weeks, the OTs might have a student for a different period of time, the nurses for a different period of time. We all start and finish at different time so it's the logistics of that." (Physiotherapist, acute, interview 16)

A low ratio of practice educators to students restricted the number of placements offered to students and thus the potential for students from more than one profession to be on placement at the same time: 
"Mentors [practice educators] are getting fewer and fewer, students are getting more and more." (Mental Health Nurse, community, interview 10) Some departments were unable to accommodate students, due to the specialised acute care needs of their service users. Participants maintained that safety, privacy and practicalities of delivering patient-centred care sometimes had to be prioritised above IPPL opportunities:

The needs [of clients in the department] are far too acute for it to be an appropriate placement for students." (Learning Disabilities Nurse, community, interview 14)

"Staff over here absolutely refuse to let anybody in the control room who don't need to be in there...if the patient's particularly unwell."

(Radiographer, acute, interview 20)

Lack of space for students and teaching space were also perceived as potential barriers to IPPL:

"It's a bit of a challenge even finding an empty room even to have a workshop with them [students]." (Dietician, community and acute, interview 19)

One of the participants highlighted that lack of time and workload priorities were potential barriers to IPPL and practice learning in general:

"Staff are just so busy, they don't have time to meet their own learning needs without spending, you know formal time teaching, providing interprofessional learning." (Midwife, acute, interview 15)Missed Opportunities for IPPL

Participants' experiences of facilitating IPPL suggested that at times the role of the student was passive, and the nature of the learning didactic. Examples given included students shadowing another professional or receiving a talk given by another professional: 
"They'll [individual students from different profession] come to clinic...and I'll tell them what my role is or whatever. So there's a lot of that goes on, but it's just you know, 'come for an hour and l'll tell you what I do'." (Dietician, acute and community, interview 19).

There was awareness that students from different professional programmes of training passed through departments on a regular basis. Opportunities for IPPL often went unrecognised or it was coincidental that students came together for learning opportunities:

"There have been times where you have ended up doing a joint visit maybe with the GP and they've had a medical student with them and we've had a nursing student but not really as a planned intervention." (District Nurse, community, interview 5)

Organisational Culture and IPPL

Organisational culture emerged as a sub-theme of barriers to IPPL.

Participants referred to the traditional culture of students learning in professional "silos" during their placements, and generally only sharing the same space with members of their own professional group:

"There is a cultural perception that medics have to learn about medicine, nurses have to learn about nursing and the social workers have to learn about social work." (GP, community, interview 4)

"When staff go for their breaks they take the students with them and they tend to sit in their own little group or profession." (Radiographer, acute and community, interview 20)

\section{Discussion}

The results from this study demonstrated that practice educators' attitudes towards IPC, and IPPL opportunities for their students were positive. In relation to IPC, these results are consistent with those of McCray (2003); Reid, Bruce, Allstaff 
and McLernon (2006); Baxter and Brumfit (2008); Colyer (2008), and Braithwaite et al. $(2012 ; 2013)$ and is further evidence that IPC is valued by practice educators. In relation to IPPL, the results are supported by recent research by Ong et al. (2017), and in previous research byReid et al. (2006) and Braithwaite et al. (2012; 2013). The context of Reid et al's. (2006) study is of particular interest, as this took place within a community care setting in Scotland, a similar context to the unit of analysis in this particular case study, and also utilised an adapted version of the RIPLS to measure attitudes. It is important to highlight that the comparisons drawn between this study and Reid et al. (2006) and Braithwaite et al. (2012; 2013) may be somewhat limited as these focussed on IPPL for qualified professionals, as opposed to IPPL for students. However, these findings provide further evidence that IPE is valued in both academic and practice settings.

D’Amour and Oandasan's (2005) framework for Interprofessional Education for Collaborative-centred Practice is a useful framework to consider the findings of this study. This framework illustrates the interplay of micro, meso and macro level factors which can affect the planning and implementation of IPE in academic settings and IPC in practice. Attitudes towards IPE and IPC are considered as examples of micro level factors; administrative processes and leadership as meso factors; and political and institutional support as macro factors (D'Amour \& Oandasan, 2005; Oandasan \& Reeves, 2005). Negative attitudes towards IPE has been reported in a number of studies as one of barriers to IPE (Freeth, Hammick, Reeves, Koppel \& Barr , 2005; Curran et al., 2007; Rees \& Johnson, 2007) but these studies have mainly focussed on IPE within academic settings. In contrast, this study, in demonstrating that attitudes towards IPC, and IPPL were positive, suggests that 
mainly meso and macro level factors impacted on the implementation of IPC, and IPPL for students' learning in practice settings.

Allport's (1954) contact theory has been considered a useful theory to understand key conditions for effective interprofessional relationships (Mohaupt et al., 2012). In this study, key conditions included close working proximity and opportunities for ad hoc communication were perceived to enable IPC, and contribute to an interprofessional team identity. Proximity as an enabler of IPC has previously been discussed by Reeves and Lewin (2004), Russell, Nyhof-Young, Abosh and Robinson (2006) and Costa, Barg, Asch and Kahn (2014). In addition, participants who identified themselves as part of an established team perceived that this enabled IPC; whereas, participants who had had their established team disrupted by restructuring of services due to health and social care integration asserted that this was initially a barrier to IPC. These findings are consistent with those of Baxter and Brumfit (2008) and Costa et al. (2014) who reported that established teams have a positive influence on interprofessional team identity. Changes in organisational structure and physical working environment can negatively affect patterns of interactions, encourage feelings of loss and grief for prior team structures (Colyer, 2008) and add to confusion around roles and responsibilities (Goldman, Meuser, Rogers, Lawrie \& Reeves, 2010).

Differences in professional governance, differing polices between health and social care, and limited access to shared systems were perceived as IPC barriers. However, there was a sense that some recent Government policies related to child protection renewed the drive for improving collaboration between professionals in health care, social work, and other agencies. As evidenced by the positive attitudes 
towards IPC found in this study, these findings suggest that health and social care professionals are driven to improve collaboration.

Although power differentials and hierarchies were discussed within the theme of organisational culture, these seemed to occur intraprofessionally and interprofessionally. This is consistent with the work of Brown et al. (2011), Lingard et al. (2012) and Powell and Davies (2012) and further reinforces social identity theory as an effective framework to explain how professionals behave and interact within organisations (Kreindler, Dowd, Star \& Gottschalk, 2012; Mitchell, Parker \& Giles, 2011; Reeves, Lewin, Espin \& Zwarenstein, 2010). Security, familiarity and confidence are evident within 'inter-group' interactions where individuals have membership in a group. Tensions can arise with 'outer-group' interactions with individuals perceived as not belonging to a group (Tajfel, 1978; Turner, 1987). The intraprofessional tensions which were apparent across teams in different care contexts, suggest that 'inter-group' and 'outer-group' conflict was evident.

As illustrated in Figure 1, it was suggested that there may be a possible link between the enablers of IPC and IPPL. In practice settings where regular IPPL occurred involving students and service users, participants described a number of IPC enablers. However, this finding is based on only three regular IPPL activities and would therefore require further investigation.

IPPL activities were limited and numerous opportunities for IPPL for students sharing the same placement at the same time were missed. The majority of activities described as IPPL suggested that the role of students was as passive learners as opposed to "active, interactive, reflective and patient centred" as recommended by Barr and Low (2013, p.19). Given the number of interview participants in the survey reporting prior experiences of IPE, it was surprising to find 
that a range of IPPL opportunities were missed. These findings suggest practice educators' misunderstanding of the concept of IPPL. The misconceptions around IPE as an educational process have previously been discussed (Reeves et al., 2011; Reeves, Perrier, Goldman, Freeth \& Zwarenstein, 2013). However, this study highlights that these misconceptions extend beyond the academic setting which suggests that further work is required to improve understanding of IPPL within practice settings.

Participants referred to the traditional culture of students learning in professional silos during their placements, and generally only sharing the same space with members of their own professional group. Previous research found that less positive attitudes towards IPPL influenced less engagement in IPPL and was also associated with doctors who were reported to have stronger professional identities (Baker, Egan-Lee, Martimianakis \& Reeves, 2011). In contrast to these findings, the qualitative findings from this study suggest that students learning in professional silos during placements may not be necessarily associated with practice educators' negative attitudes towards IPPL but more associated with some of the practical and logistical challenges to planning and implementing IPPL in practice settings.

The insight that this study gave into the perspectives of practice educators working in a variety of care settings highlighted some barriers which may be unique to practice settings. Participants from specialised areas such as learning disabilities and mental health highlighted that the presence of one student, let alone an interprofessional mix of students, was at times inappropriate in one to one consultations or with service users deemed vulnerable. These findings build on the 
work of Stew (2005) and Pollard (2009) who reported that opportunities for IPPL may depend on situational and contextual factors.

\section{Limitations}

This study used two pre-validated questionnaires to measure attitudes towards IPC and IPPL. However, for the purposes of this this study, the scales were combined, and a 4-point Likert scale response was adopted. Although intrarater reliability was measured using a test-retest method, the validity of the survey was not measured.

In analysing demographic data generated from the survey, the majority of survey participants reported that they had prior experience of IPE. However, on interviewing participants, some of the experiences that participants described were multiprofessional or shared learning, rather than interprofessional learning. Further inquiry into the nature of participants' prior IPE experience may have enabled a more reliable analysis of the effect of this variable on attitudes towards IPPL.

In identifying the most appropriate methods of data generation for the qualitative data in this study, the limitations of semi-structured interviews were considered. For example, face to face semi-structured interviews can be time consuming and the presence of the researcher may inhibit or influence discussion (Ward, Gott \& Hoare (2015). Although the interviews were time consuming, being able to enter the participants' workplace in this study, provided the researcher with a rich insight into their working environment and contributed to understandings of the enablers and barriers to IPC and IPPL.

As a single case study, these limitations may add to the scepticism around case studies and concerns regarding the generalisability of its findings (Yin, 2014). 
However, in relation to IPC, these findings contribute to existing knowledge and offer new evidence during a time in health and social care where major changes require professionals within these services to change the way that they work together. In relation to IPPL, given that there are few other studies that have investigated the attitudes and perceptions of practice educators' (from more than one professional group), these findings contribute to the understanding of how IPPL for students is valued. This knowledge can help with identifying strategies for improving IPC and strengthening the continuum of IPE from academic settings to practice settings.

\section{Concluding Comments}

As a mixed-methods case study, the findings are instrumental in gaining insight into practice educators' attitudes towards IPC and IPPL, and the enablers and barriers to these within practice. This study has demonstrated that health care and social work practice educators have positive intentions to work interprofessionally and are supportive of students learning together within practice settings. Although attitudes were positive, there were some factors which impacted on implementation of IPC and IPPL.

The findings of this study make an important contribution to existing knowledge and future practice and policy related to IPC and IPPL. Given that there are few other studies that have investigated practice educators' attitudes and perspectives of both IPC and IPPL, these findings contribute to the understanding of how these are valued in practice settings. This knowledge can help identify strategies for improving IPC, strengthen the identity of interprofessional teams, and enhance IPPL opportunities for students. 


\section{Acknowledgements}

The authors would like to acknowledge and thank Fidan Fraser and Katherine

Paramore for their research assistance.

\section{Declaration of interest}

The authors report no declarations of interest. The authors were responsible for the writing and content of this paper.

\section{References}

Adams, A., Bond, S., \& Arber, S. (1995) Development and validation of scales to measure organizational features of acute hospital wards. International Journal of Nursing Studies, 32, 6, 612-627. (doi: 10.1016/00207489(95)00041-1)

Allport G, W. (1954). The nature of prejudice. New York: Doubleday \& Company Inc

Bailey, P., Jones, L., \& Way, D. (2006). Family physician/nurse practitioner: Stories of collaboration. Journal of Advanced Nursing, 53, 381-391. (doi: 10.1111/j.1365-2648.2006.03734.x)

Baker, L., Egan-Lee, E., Martimianakis, M.A., \& Reeves, S. (2011). Relationships of power: implications for interprofessional education. Journal of Interprofessional Care, 25, 98-104. (doi: https://doi.org/10.1111/j.13652648.2006.03734.x)

Barr, H. \& Low, H. (2013). Introducing interprofessional education. Fareham, UK: CAIPE

Baxter, S. K., \& Brumfitt, S. M. (2008). Professional differences in interprofessional working. Journal of Interprofessional Care, 22, 239-251.

(doi: 10.1080/13561820802054655)

Braithwaite, J., Westbrook, M., Nugus, P., Greenfield, D., Travaglia, J., Runciman, W., .. . Westbrook, J. (2012). A four-year, systems-wide intervention promoting interprofessional collaboration. BMC Health Services Research, 12, 99. (doi: 10.1186/1472-6963-12-99)

Braithwaite, J., Westbrook, M., Nugus, P., Greenfield, D., Travaglia, J. Runciman, W., ... Westbrook. (2013) Continuing differences between health professions' attitudes: The saga of accomplishing systems-wide interprofessionalism. International Journal for Quality in Health Care, 25, 8-15. (doi:10.1093/intahc/mzs071) 
Brown, J., Lewis, L., Ellis, K., Stewart, M., Freeman, T.R. \& Kasperski, M.J. (2011). Conflict on interprofessional primary health care teams - can it be resolved? Journal of Interprofessional Care, 25, 4-10. (doi: 10.3109/13561820.2010.497750)

CAIPE. (2002). Interprofessional education: A definition. London: CAIPE.

Children and Young People (Scotland) Act 2014, Crown copy right. The Stationery Office Limited, UK. Retrieved from http://www.legislation.gov.uk/asp/2014/8/contents/enacted

Colyer, H. M. (2008). Embedding interprofessional learning in pre-registration education in health and social care: Evidence of cultural lag. Learning in Health and Social Care, 7,126-133. (doi: 10.1111/j.1473-6861.2008.00185.x)

Costa, D.K., Barg, F.K., Asch, D.A., \& Kahn, J.M. (2014). Facilitators of an interprofessional approach to care in medical and mixed medical/surgical ICUs: A multicentre qualitative study. Research in Nursing \& Health, 37, 326335. (doi:10.1002/nur.21607)

Creswell, J.W. (2015). A concise introduction to mixed methods research. Thousand Oaks, CA: Sage Publications, Inc.

Curran, V.R., Sharpe, D. \& Forristall, J. (2007). Attitudes of health sciences faculty members towards interprofessional teamwork and education. Medical Education, 41, 9, 892-896. (doi: 10.1111/j.1365-2923.2007. 02823.x)

D'Amour, D. \& Oandasan, I. (2005). Interprofessionality as the field of interprofessional practice and interprofessional education: An emerging concept. Journal of Interprofessional Care, Supplement 1, 8-20 (doi: 1080/13561820500081604)

Freeth, D., Hammick, M., Reeves, S., Koppel, I. \& Barr, H. (2005). Effective interprofessional education: development, delivery and evaluation. Oxford, Blackwell Publishing Ltd.

Gerring, J. (2007). Case study research. Principles and Practices. New York: Cambridge University Press.

Gillham, B. (2010). Case study research methods. London: Continuum International Publishing.

Goldman, J., Meuser, J., Rogers, J., Lawrie, L. \& Reeves, S. (2010). Interprofessional collaboration in family health teams. Canadian Family Physician, 56, 368-74

Institute of Medicine. (2015). Measuring the impact of interprofessional education on collaborative practice and patient outcomes. Retrieved from htto://www.nap.edu.

Kenaszchuk, C., Reeves, S., Nicholas, D., \& Zwarenstein, M. (2010). Validity and reliability of a multi-group measurement scale for interprofessional collaboration. Biomedical Central Health Services Research, 10, 83. 
Kreindler, S, A., Dowd, D, A., Star, N, D. \& Gottschalk, T. (2012) Silos and social identity: The social identity approach as a framework for understanding and overcoming divisions in health care. The Milbank Quarterly, 90, 347-374. (doi: 10.1111/j.1468-0009.2012.00666.x)

Lingard, L., McDougall, A., Levstik, M., Chandok, N., Spafford, M. M., \& Schryer, C. (2012). Representing complexity well: A story about teamwork, with implications for how we teach collaboration. Medical Education, 46, 869-877. (doi: 10.1111/j.1365-2923.2012.04339.x)

McCray, J. (2003). Interprofessional practice and learning disability nursing. British Journal of Nursing, 12,1335-1344. (doi: 10.12968/bjon.2003.12.22.11897)

Mitchell, R.J., Parker, V. \& Giles, M. (2011). When do interprofessional teams succeed? Investigating the moderating roles of team and professional identity in interprofessional effectiveness. Human Relations, 64,1321-1343. (doi: $10.1177 / 0018726711416872$ )

Mohaupt, J., van Soeren, M., Andrusyszyn, M., MacMillan,K., Devlin-Cop, S \& Reeves, S. (2012) Understanding interprofessional relationships by the use of contact theory, Journal of Interprofessional Care, 26, 370375. (doi: 10.3109/13561820.2012.673512)

Oandasan, I. \& Reeves, S. (2005). Key elements of interprofessional education. Part 2: Factors, processes and outcomes. Journal of Interprofessional Care, 19:sup1, 39-48. (doi: 10.1080/13561820500081703)

O'Carroll, V. (2017). Practice Mentors' Attitudes and Perspectives of Interprofessional Working, and Interprofessional Practice Learning for Students: A Mixed-Methods Case Study (Unpublished Doctoral thesis). Available from http://hdl.handle.net/10023/10482

O'Carroll, V., Braid, M., Jackson, C. M. \& Ker, J. (2012). How can student experience enhance the development of a model of interprofessional clinical skills education in the practice placement setting? Journal of Interprofessional Care, 26, 508-510. (doi: 10.3109/13561820.2012.709202)

O'Carroll, V., McSwiggan, L. \& Campbell, M. (2016). Health and social care professionals' attitudes to interprofessional working and interprofessional education: A literature review. Journal of Interprofessional Care, 30, 42-49. (doi: 10.3109/13561820.2015.1051614)

O'Carroll,V., Smith,J., Collinson, A., Jackson, C., \& Ker, J. (2013). Interprofessional education for students' in primary care placements: Widening opportunities for accessible interprofessional learning. Medical Science Educator, 23 (special issue), 405-410. (doi: 10.1007/BF03341656)

Ong, S.K., Tan, N.C.K., Knab, M.S., Farrell S.E. \& Lim, W.S. (2017). Attitudes of clinician educators towards interprofessional education and collaboration: Insights from two interprofessional scales. Journal of Interprofessional Care, 31,1-5. (doi: 10.1080/13561820.2017.1320275) 
Parsell, G., \& Bligh, J. (1999). The development of a questionnaire to assess the readiness of health care students for interprofessional learning (RIPLS). Medical Education, 33, 095-100.

Pollard, K. (2009) Student engagement in interprofessional working in practice placement settings. Journal of Clinical Nursing, 8, 2846-56. (doi: 10.1111/j.1365-2702.2008.02608)

Pollard, K. C., Miers, M. E., \& Rickaby, C. (2012). “Oh why didn't I take more notice?" Professionals' views and perceptions of pre-qualifying preparation for interprofessional working in practice. Journal of Interprofessional Care, 26, pp.355-361. (doi: 10.3109/13561820.2012.689785)

Ponzer, S., Hylin, U., Kusoffsky, A., Lauffs, M., Lonka, K., Mattiasson, A.C., \& Nordström, G. (2004). Interprofessional training in the context of clinical practice: goals and student's perception on clinical education wards. Medical Education, 38,727-736. (doi:10.1111/j.1365-2929.2004.01848.x)

Powell, A.E. \& Davies, H.T.O. (2012). The struggle to improve patient care in the face of professional boundaries. Social Science and Medicine, 75, 807-814. (doi: 10.1016/j.socscimed.2012.03.049)

Reid, R., Bruce, D., Allstaff, K., \& McLernon, D. (2006). Validating the readiness for interprofessional learning scale (RIPLS) in the postgraduate context: Are health care professionals ready for IPL? Medical Education, 40, 415-422. (doi: 10.1111/j.1365-2929.2006.02442.x)

Rees, D., \& Johnson, R. (2007). All together now? Staff views and experiences of a pre-qualifying interprofessional curriculum. Journal of Interprofessional Care, 21, 543-555. (doi: 10.1080/13561820701507878)

Reeves, S., Goldman, J., Gilbert, J., Tepper, J., Silver, I., Suter, E., \& Zwarenstein, M., (2011). A scoping review to improve conceptual clarity of interprofessional interventions. Journal of Interprofessional Care. 25, 167174. (doi: $10.3109 / 13561820.2010 .529960)$

Reeves, S., \& Lewin, S. (2004). Interprofessional collaboration in the hospital: Strategies and meanings. Journal of Health Services Research and Policy, 9, 218-225. (doi: 10.1258/1355819042250140)

Reeves, S., Lewin, S., Espin, S. \& Zwarenstein, M. (2010). Interprofessional teamwork for health and social care. West Sussex: Blackwell Publishing Ltd,

Reeves, S., Perrier, L., Goldman, J., Freeth, D., \& Zwarenstein, M. (2013). Interprofessional education: Effects on professional practice and healthcare outcomes (Update). The Cochrane Library. Issue 3, West Sussex, UK: John Wiley \& Sons, Ltd. (doi: 10.1002/14651858.CD002213.pub3)

Ritchie, J. \& Spencer, L (1994). Qualitative data analysis for applied policy research. In A. Bryman \& R. Burgess (Eds.), Analysing qualitative data (pp.173-194). London: Routledge. 
Russell, L., Nyhof-Young, J., Abosh, B., \& Robinson, S. (2006). An exploratory analysis of an interprofessional learning environment in two hospital clinical teaching units. Journal of Interprofessional Care, 20, 29-39. (doi: 10.1080/13561820500476473)

Stake, R.E. (1995). The art of case study research. London: Sage Publications Ltd.

Stew, G. (2005). Learning together in practice: a survey of interprofessional education in clinical settings in South-East England. Journal of Interprofessional Care, 19, 223-235. (doi: 10.1080/13561820500138685)

Sullivan-Bollyai, S. \& Grey, M. (2002). Data collection methods. In G. LoBiondoWood \& J. Haber (Eds.), Research methods, critical appraisal and utilization. (pp.293-310). Missouri: Mosby, Inc.,

Tajfel, H. (1978). Social categorization, social identity and social comparison. In H. Tajfel (Ed.) Differentiation between social groups (pp. 61-76). London: Academic Press Inc

Turner, J.C. (1987). Rediscovering the social group. Oxford: Basil Blackwell Ltd.

Ward, K., Gott, M. \& Hoare, K. (2015). Participants' views of telephone interviews within a grounded theory study. Journal of Advanced Nursing, 71, 12, 2775-2785.

World Health Organisation. (2010) Framework for action on interprofessional education and collaborative practice. WHO, Switzerland. Retrieved from http://www.who.int/hrh/nursing_midwifery/en/.

Yin, R.K. (2014). Case study research. Design and methods. (3rd ed.) Thousand Oaks, CA: Sage Publications, Inc. 